\title{
Erratum to: Implementation and verification of a masonry panel model for nonlinear dynamic analysis of infilled RC frames
}

\author{
E. Smyrou - C. Blandon - S. Antoniou - R. Pinho • \\ F. Crisafulli
}

Published online: 16 September 2011

(C) Springer Science+Business Media B.V. 2011

\section{Erratum to: Bull Earthquake Eng DOI 10.1007/s10518-011-9262-6}

Unfortunately the authors' affiliations for this paper were either incomplete or incorrect. They should have been as follows:

E. Smyrou $(\varangle) \cdot$ C. Blandon · R. Pinho

Rose School, IUSS, University of Pavia, Pavia, Italy

e-mail: smiroulena@gmail.com

S. Antoniou

SeismoSoft—Software Solutions for Earthquake Engineering, Chalkis, Greece

F. Crisafulli

Universidad Nacional de Cuyo, Mendoza, Argentina

The online version of the original article can be found under doi:10.1007/s10518-011-9262-6.

E. Smyrou $(\varangle) \cdot$ C. Blandon · R. Pinho

Rose School, IUSS, University of Pavia, Pavia, Italy

e-mail: smiroulena@gmail.com

S. Antoniou

SeismoSoft-Software Solutions for Earthquake Engineering, Chalkis, Greece

F. Crisafulli

Universidad Nacional de Cuyo, Mendoza, Argentina 